\title{
Microtopographic non-invasive inspection for determining roughness parameters of dental biomaterials
}

\section{Inspección microtopográfica no invasiva para la determinación de parámetros de rugosidad de biomateriales dentales}

\author{
Alicia Fernández-Oliveras $\left(1, \mathrm{~S},{ }^{*}\right)$, Manuel F. M. Costa(2), María del Mar Pérez ${ }^{(3, S)}$
}

1. Departamento de Didáctica de las Ciencias Experimentales, Facultad de Ciencias de la Educación, Universidad de Granada, Campus de Cartuja s/n. 18071 Granada, Spain.

2. Centro de Física, Universidade do Minho, Campus de Gualtar, 4710-057 Braga, Portugal.

3. Departamento de Óptica, Facultad de Ciencias, Universidad de Granada. Edificio Mecenas, Campus Fuentenueva s/n. 18071 Granada, Spain.

(*) Email: alilia@ugr.es

S: miembro de SEDOPTICA / SEDOPTICA member

Received / Recibido: 16/12/2013. Revised / Revisado: 05/04/2014. Accepted / Aceptado: 10/04/2014.

DOI: http://dx.doi.org/10.7149/OPA.47.2.131

\begin{abstract}
:
In this work we experimentally analyze the roughness parameters of two types of dental-resin composites and pre-sintered and sintered zirconia ceramics. We studied two shades of both composite types and two sintered zirconia ceramics: colored and uncolored. A surface treatment was applied to one specimen of each dental-resin. The samples were submitted to non-invasive inspection with the MICROTOP.06.MFC laser microtopographer to gather meaningful surface statistical parameters. For a comparison of the different biomaterials, the uncertainties associated to the surface parameters were also determined. Except for the composites with the surface treatment, the sample surfaces had approximately a normal distribution of heights.
\end{abstract}

Key words: Roughness Parameters, Microtopography, Non-Invasive Inspection, Biomaterials, Dental-Resin Composites, Zirconia Ceramics.

\section{RESUMEN:}

En este trabajo se analizan experimentalmente los parámetros de rugosidad de dos tipos de resinas de composite (material compuesto) dentales y de cerámicas de circonia presinterizada y sinterizada. Se estudiaron dos tonalidades (shades) de ambos composites y cerámicas sinterizadas con y sin color. Se aplicó un tratamiento superficial a un espécimen de cada resina. Las muestras fueron inspeccionadas de forma no invasiva empleando el microtopógrafo láser MICROTOP.06.MFC para obtener los principales parámetros estadísticos de la superficie. Para comparar los distintos biomateriales, se determinaron también las incertidumbres asociadas. Salvo para los composites con el tratamiento superficial, las superficies de las muestras presentaron aproximadamente una distribución normal de alturas.

Palabras clave: Parámetros de Rugosidad, Microtopografía, Inspección no Invasiva, Resinas de Composite Dentales, Cerámicas de Circonia.

\section{REFERENCES AND LINKS / REFERENCIAS Y ENLACES}

[1]. M. Quirynen, M. Marechal, H. J. Busscher, A. H.Weerkamp, P. L. Darius, D. Van Steenberghe, "The influence of surface free energy and surface roughness on early plaque formation. An in vivo study in man", J. Clin. Periodontol. 17, 138-144 (1990). DOI

[2]. M. Quirynen, H. C. van der Mei, C. M. Bollen, A. Schotte, M. Marechal, G. I. Doornbusch, I. Naert, H. J. Busscher, D. Steenberghe, "An in vivo study of the influence of the surface roughness of implants on the microbiology of supra- and subgingival plaque”, J. Dent. Res. 72, 1304-1309 (1993). DOI 
[3]. C. Wu-Yuan, K. Eganhouse, J. Keller, K. Walters, “Oral bacterial attachment to titanium surfaces: a scanning electron microscopy study", J. Oral Implantol. 21, 207-213 (1995).

[4]. C. Bollen, W. Papaioannou, J. Van Eldere, E. Scheppers, M. Quirynen, D. Van Steenberghe, "The influence of abutment surface roughness on plaque accumulation and peri-implant mucositis", Clin. Oral Implant. Res. 7, 201-211 (1996). DOI

[5]. M. Quirynen, C. Bollen, W. Papaioannou, J. Van Eldere, D. Van Steenberghe, "The influence of titanium abutments surface roughness on plaque accumulation and gingivitis. Short term observations", Int. J. Oral Maxillofac. Implants. 11, 169-178 (1996).

[6]. S. B. Mitra, D. Wu, B. N. Holmes, "An application of nanotechnology in advanced dental materials", J. Am. Dent. Assoc. 134, 1382-1390 (2003). DOI

[7]. J. Denrya, R. Kellyb, "State of the art of zirconia for dental applications", Dent. Mater. 24, 299-307 (2008). DOI

[8]. J. Chevalier, L. Gremillard, "Ceramics for medical applications: A picture for the next 20 years", J. Eur. Ceram. Soc. 29, 1245-1255 (2009). DOI

[9]. A. Fernández-Oliveras, M. Rubiño, M. M. Pérez, "Scattering and absorption properties of biomaterials for dental restorative applications", J. Eur. Opt. Soc. Rap. Public. 8, 13056 (2013). DOI

[10]. A. Fernández-Oliveras, M. Rubiño, M. M. Pérez, "Análisis comparativo de medidas de anisotropía de scattering en tejidos y biomateriales dentales", Opt. Pura Apl. 46, 183-188 (2013). DOI

[11]. A. Fernández-Oliveras, M. Rubiño, M. M. Pérez, "Scattering anisotropy measurements in dental tissues and biomaterials", J. Eur. Opt. Soc. Rap. Public. 7, 12016 (2012). DOI

[12]. M. F. M. Costa, "Optical triangulation-based microtopographic inspection of surfaces”, Sensors 12, 4399-4420 (2012). DOI

[13]. M. C. L. Pacheco, M. F. M. Costa, A. J. P. Zapata, J. D. Cherit, E. R. Gallegos, "Implementation and analysis of relief patterns of the surface of benign and malignant lesions of the skin by microtopography", Phys. Med. Biol. 50, 5535-5543 (2005). DOI

[14]. M. F. M. Costa, P. B. Pereira, "Optical microtopographic inspection of the surface of tooth subjected to stripping reduction", Proc. SPIE 8001, 80012X (2011). DOI

[15]. M. F. M. Costa, "Surface Inspection by an optical triangulation method", Opt. Eng. 35, 2743-2747 (1996). DOI

[16]. M. F. M. Costa, V. Teixeira, "Rugometric and Microtopographic Inspection of $\mathrm{Cr}^{-} \mathrm{Cr}_{2} \mathrm{O}_{3}$ cermet solar absorbers", Int. J. Photoenergy 1110-662X, 82327 (2007). DOI

[17]. M. F. M. Costa, C. Batista, A. Portinha, V. Teixeira, C. R. Oliveira, R., Ribeiro, “Microtopographic inspection of laser glazed thermal barrier coatings", Opt. Eng. 47, 063603 (2008). DOI

[18]. International Organization for Standardization, Guide to the Expression of Uncertainty in Measurement. Corrected and reprinted (Geneva, 1995).

\section{Introducción}

La evaluación de las propiedades de la superficie de nuevos biomateriales tiene una importante repercusión de cara a sus aplicaciones biomédicas. En odontología, estudios en vivo y en vitro han demostrado que existe una relación significativa entre la textura de la superficie y la formación de placa [1-3].

Entre los factores que permiten la adhesión de bacterias, la rugosidad de la superficie es el factor de mayor influencia, de modo que una rugosidad inferior se corresponde con una menor retención de placa [4,5]. Además, una mayor rugosidad del la superficie dental favorece la penetración de bacterias y conduce a un mayor riesgo de caries. Por ello, en odontología restauradora, es fundamental el estudio de las propiedades de la superficie de los biomateriales destinados a sustituir tejidos dentales dañados de forma irreversible para evitar cambios perjudiciales en tratamientos futuros.

Actualmente, los materiales más ampliamente utilizados en la restauración del esmalte dental son las resinas de composite (material compuesto). Entre ellas, aquellas con nanorelleno [6] pueden tener propiedades distintas a las resinas con rellenos tradicionales (híbridos y 
supraesféricos) por ser sus partículas de relleno de escala nanométrica. Por otro lado, un material que se utiliza actualmente para restaurar la dentina es la cerámica de circonia en su forma policristalina tetragonal y dopada con itrio (3YTZP) $[7,8]$.

El uso en odontología de estos biomateriales se ha extendido a tenor de sus buenas propiedades biológicas y mecánicas. Sus propiedades ópticas se han analizado en trabajos anteriores [9-11], pero sus características rugométricas aún no han sido estudiadas en profundidad. En este sentido, se hace necesaria la evaluación de los principales parámetros de rugosidad en resinas de composite y cerámicas de circonia proporcionando información, no sólo sobre la rugosidad media, sino también sobre la distribución superficial de alturas. Además, es de gran interés establecer comparaciones entre diversos materiales y distinciones entre un mismo material en diferentes condiciones (por ejemplo, antes y después del tratamiento superficial en composites o del proceso de sinterización en cerámicas de circonia).

Un método adecuado para tal fin debe permitir obtener los valores de los parámetros estadísticos de la superficie con la precisión suficiente para encontrar diferencias significativas. Ello solo puede comprobarse conociendo la incertidumbre asociada a los valores de los parámetros de rugosidad obtenidos. Esto se obvia de forma generalizada en los trabajos que estudian biomateriales y, concretamente, biomateriales destinados a aplicaciones dentales, de modo que no es posible conocer la precisión de los valores facilitados, ni en qué medida las comparaciones establecidas se basan de diferencias significativas. Incluso proporcionando la incertidumbre asociada, ha de tenerse en cuenta que el valor de la rugosidad depende del método de medida empleado y de la superficie examinada.

La creciente diversidad de tipos de superficies, las limitaciones de medida y los exigentes requerimientos de tolerancia han demandado un importante esfuerzo en el desarrollo de distintos métodos, sistemas y técnicas metrológicas para la evaluación de superficies. Los métodos ópticos juegan un papel primordial en la inspección no invasiva. Entre estos métodos, los basados en triangulación óptica han adquirido un estatus importante merced a su flexibilidad, fiabilidad y robustez. La triangulación óptica ha demostrado ser una herramienta muy valiosa para la evaluación microtopográfica de superficies en los ámbitos industrial y científico [12]. Concretamente, en el campo de las ciencias de la vida se han desarrollado diversas aplicaciones con éxito $[13,14]$.

El objetivo de este trabajo es analizar experimentalmente y comparar los parámetros de rugosidad característicos de la superficie de distintos tipos de resinas de composite y de cerámicas de circonia destinadas a aplicaciones dentales. Con este fin, se llevó a cabo la inspección microtopográfica no invasiva de dichos biomateriales empleando el sistema basado en triangulación óptica activa MICROTOP.06.MFC.

\section{Materiales y método}

\section{2.a. Preparación de las muestras}

Se analizaron dos tipos distintos de composites dentales: uno con relleno supraesférico (composite supraesférico) y otro con nanorelleno (nanocomposite). Ambos tipos de resinas de composites poseían matrices poliméricas similares, compuestas de dimetacrilatos. Las características de los dos tipos de resinas de composite dental analizadas, de acuerdo con los datos de los fabricantes, se muestran en la Tabla I.

Cada muestra de composite se fabricó en una placa de vidrio de $1 \mathrm{~mm}$ de espesor (Knittel GLASER, Bielefeld, Germany) en la que se preparó un orificio circular en el que introdujo la resina de composite. Tras colocar la resina, tanto en la parte superior como en la inferior, se dispuso una lámina de plástico transparente (Acrylite Plus Clear, Tap Plastics, Dublin, CA, USA). Encima se colocó otra placa de vidrio para homogenizar el espesor de la muestra. Cada muestra se fotopolimerizó durante 40 segundos utilizando una unidad de fotopolimerización (Bluephase, Ivoclar Vivadent AG, Liechtenstein) con una irradiancia de $1100 \mathrm{~mW} / \mathrm{cm}^{2} \pm 10 \%$. 


\section{TABLA I}

Características de los dos tipos de resinas de composite dental analizadas según datos de los fabricantes.

\begin{tabular}{|c|c|c|c|}
\hline $\begin{array}{l}\text { Resina } \\
\text { Dental }\end{array}$ & $\begin{array}{c}\text { Matriz } \\
\text { Orgánica }\end{array}$ & $\begin{array}{c}\text { Relleno } \\
\text { Inorgánico }\end{array}$ & Tipo \\
\hline $\begin{array}{c}\text { Filtek } \\
\text { Supreme } \\
\text { XT }\end{array}$ & $\begin{array}{c}\text { Bis-GMA, } \\
\text { Bis-EMA, } \\
\text { UDMA, } \\
\text { TEGDMA }\end{array}$ & $\begin{array}{c}\text { Sílice } \\
\text { aglomerado } \\
\text { y sílice } \\
\text { altamente } \\
\text { dispersado }\end{array}$ & Nanocomposite \\
\hline $\begin{array}{c}\text { Tetric } \\
\text { EvoCeram }\end{array}$ & $\begin{array}{l}\text { Bis-GMA, } \\
\text { TEGDMA }\end{array}$ & $\begin{array}{l}\text { Sílice, óxido } \\
\text { de zirconio }\end{array}$ & $\begin{array}{l}\text { Composite } \\
\text { supraesférico }\end{array}$ \\
\hline
\end{tabular}

Los especímenes obtenidos tras el procedimiento descrito se denominan Mylar strip. Después de la fotopolimerización, se aplicó el tratamiento superficial OptiDisc ${ }^{\circledR}$ de Kerr a un espécimen de cada tipo de resina de composite dental. En este tratamiento superficial, se emplea una secuencia de cuatro discos abrasivos y, por consiguiente, se espera que la rugosidad resultante sea mayor que en las superficies sin el tratamiento.

Aunque las muestras fueron polimerizadas sobre un sustrato completamente plano, en la obtención de los parámetros de rugosidad, se realizó la correspondiente corrección sustrayendo el efecto de la pendiente media de la superficie en el área examinada.

Las muestras de resina dental se manipularon de acuerdo con las instrucciones de los fabricantes y fueron preparadas por la misma persona a fin de minimizar su variabilidad y hacer viables las comparaciones entre especímenes.

De ambos tipos de resinas de composite se estudiaron dos tonalidades (shades) distintos: translúcido claro y esmalte A2, para el nanocomposite, y translúcido y esmalte $\mathrm{A} 2$, en el caso del composite supraesférico.

En el análisis de la cerámicas de circonia para aplicaciones dentales, se emplearon muestras presinterizadas y sinterizadas de LAVATMZirconia proporcionadas por el fabricante con espesores similares $(0,48 \mathrm{~mm}$ para las muestras presinterizadas y $0,50 \mathrm{~mm}$ para las sinterizadas). La circonia sinterizada se obtiene a partir de láminas de material presinterizado mediante procesos de diseño y manufactura asistidos por ordenador (CAD/CAM).

Se estudiaron dos muestras de cerámica de circonia sinterizada distintas: con color SP3 y sin color.

\section{2.b. Inspección microtopográfica no invasiva}

El dispositivo experimental empleado para realizar la inspección microtopográfica no invasiva de las muestras se representa en la figura 1. Se trata del microtopógrafo láser basado en triangulación óptica activa MICROTOP.06.MFC [15-17].

Este tipo de sistema de inspección de superficies se puede aplicar, no sólo en un rango de detección de larga distancia, sino también en superficies y muestras pequeñas, con resoluciones de altura de hasta $1 \mathrm{~nm}$. Permite realizar mediciones de espesores y reconstruir mapas de topografía de la superficie, además de determinar los parámetros estadísticos de caracterización de la rugosidad [15]. El montaje es muy versátil $\mathrm{y}$ ha sido utilizado para diferentes tareas de inspección en diversos tipos de muestras (películas delgadas, moldes y tejidos) [12]. Concretamente, se han realizado con éxito medidas tanto en materiales opacos como translúcidos $[13,14,16,17]$. Para la realización de las medidas, el aparato dispone de un sistema de aislamiento de vibraciones.

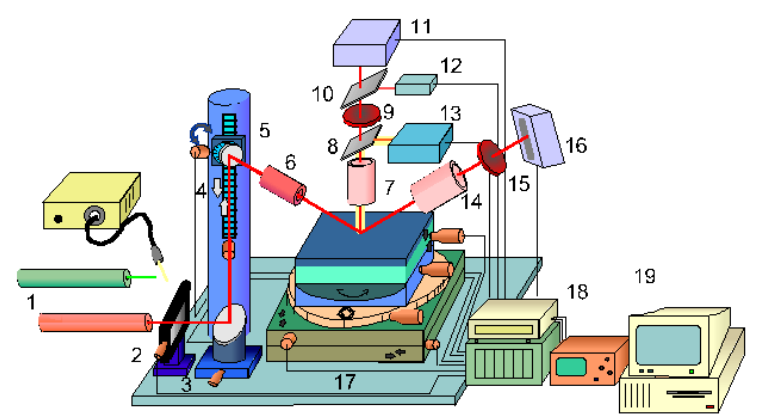

Fig. 1. Microtopógrafo laser MICROTOP.06.MFC: 1. Fuentes laser intercambiables, 2. Soporte para el aislamiento de vibraciones, 3. Filtro de densidad neutra, 4. Sistema de orientación del haz, 5. Sistema motorizado de control del ángulo de incidencia, 6. Óptica de incidencia, 7. Óptica de observación normal, 8. y 10. Divisores de haz, 9. y 15. Filtros interferenciales, 11. Sistema fotosensitivo de detección normal, 12. Fotodetector, 13. Videocamara, 14. Óptica de observación especular, 16. Sistema fotosensitivo de detección especular, 17. Soporte de la muestra y sistema de posicionamiento motorizado, 18. Sistema de control y adquisición de datos, 19. Ordenador. 
El dispositivo de triangulación utilizado opera con incidencia oblicua, realiza un barrido sincronizado punto a punto de la superfície de la muestra e incorpora sistemas de observación tanto en la dirección normal como en la especular. Los sistemas de orientación y control motorizado son los responsable de que el haz de la fuente láser incida oblicuamente sobre la muestra mientras que el barrido de la superficie se lleva a cabo mediante el sistema de posicionamiento motorizado del que consta el soporte de la muestra.

En el esquema de la Fig. 2 se representa de forma simplificada la geometría de triangulación en la que se basa el microtopógrafo laser empleado.

El área inspeccionada en cada muestra, fue 1 $\mathrm{mm}^{2}$. En esa área, se registraron 51 perfiles unidimensionales con 601 puntos por perfil, a partir de los cuales se determinaron los parámetros estadísticos de la superficie o parámetros de rugosidad de cada muestra analizada. En la determinación de dichos parámetros, se sustrajo previamente el efecto de la pendiente media de la superficie en el área inspeccionada.

Empleando el sistema MICROTOP.06.MFC se determinaron los parámetros de rugosidad esenciales, como son la rugosidad media Ra, definida como el promedio de las desviaciones absolutas respecto de la media de alturas de la superficie,

$$
R_{a}=\frac{1}{N} \sum_{i=1}^{N}\left|\Delta Z_{i}\right|,
$$

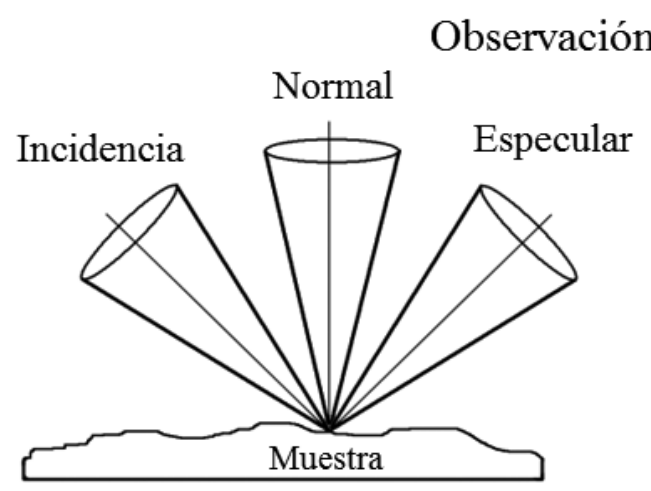

Fig. 2. Esquema de la geometría de triangulación óptica en la que se basa el microtopógrafo laser MICROTOP.06.MFC. donde $\Delta Z_{i}=Z_{i}-Z_{m}$ son las desviaciones con respecto a la altura media, $Z_{m}$ :

$$
Z_{m}=\frac{1}{N} \sum_{i=1}^{N} Z_{i},
$$

y la desviación típica $R_{q}$,

$$
R_{q}=\sqrt{\frac{1}{N} \sum_{i=1}^{N} \Delta Z_{i}^{2} .}
$$

Asimismo se determinaron otros dos parámetros estadísticos que, a pesar de que proporcionan información relevante sobre la distribución de alturas de la superficie, se obvian en la mayoría de los trabajos que analizan materiales dentales. Se trata los parámetros skewness, $R_{s k}$, definido como el tercer momento respecto de la media,

$$
R_{s k}=\frac{1}{N R_{q}^{3}} \sum_{i=1}^{N} \Delta Z_{i}^{3} .
$$

y kurtosis, $R_{k u}$, definido como el cuarto momento respecto de la media,

$$
R_{k u}=\frac{1}{N R_{q}^{4}} \sum_{i=1}^{N} \Delta Z_{i}^{4} .
$$

El valor del parámetro $R_{s k}$ varía entre menos infinito y más infinito y se relaciona con la simetría de la distribución. Para una distribución perfectamente simétrica el valor del parámetro $R_{s k}$ es cero. Una distribución con una cola asimétrica extendiéndose hacia la izquierda presentará valores negativos de dicho parámetro, mientras que una distribución con una cola asimétrica que se extiende hacia la derecha presentará valores positivos de $R_{s k}$.

El parámetro $R_{k u}$ se relaciona con la forma de la distribución. Tomando como referencia la distribución normal, que se denomina mesocúrtica $\left(R_{k u}=3\right)$, una distribución puede ser leptocúrtica, si presenta una forma más apuntada que la de la distribución normal $\left(R_{k u}>3\right)$ o platicúrtica, si presenta una forma más aplanada que la de la distribución normal $\left(R_{k u}<3\right)$.

Así, el análisis conjunto de los valores de estos dos últimos parámetros estadísticos permite evaluar si la distribución de alturas estudiada se 
asemeja a una distribución normal, para la cual se verifica que $R_{s k}=0$ y $R_{k u}=3$.

Las medidas se realizaron bajo condiciones de repetibilidad: con cada muestra el procedimiento de medida se repitió 20 veces y a partir de las 20 medidas se calculó el valor medio de cada parámetro de rugosidad y su correspondiente incertidumbre tipo $\mathrm{A}$, expresada como desviación estándar [18].

\section{Resultados y discusión}

Los valores de los parámetros de superficie obtenidos para los distintos biomateriales dentales analizados, así como sus correspondientes incertidumbres, se muestran en la Tabla II. Puede apreciarse que, como consecuencia del cálculo de incertidumbres anteriormente descrito (tipo A), las precisiones correspondientes a cada muestra son diferentes.

Puede observarse que las diferencias entre las dos tonalidades de ambos tipos de composites son significaticas. Para cada tipo de resina dental, la tonalidad esmalte A2 presenta menores valores de $R_{a}$ y $R_{q}$ que el translúcido. Estas diferencias podrían deberse a la existencia de distintas concentraciones de las partículas de relleno en las distintas tonalidades.

Comparando ambos tipos de composites del mismo shade, se encuentra que los parámetros de rugosidad $\mathrm{Ra}$ y $\mathrm{Rq}$ presentan valores superiores para el nanocomposite. Este comportamiento podría relacionarse con la presencia de cúmulos (clusters) de mayor tamaño.

TABLA II

Valores medios de los parámetros de rugosidad con sus incertidumbres asociadas, para los diferentes biomateriales dentales analizados.

\begin{tabular}{|c|c|c|c|c|c|}
\hline Biomaterial & Shade o color & $R_{a}$ medio $(\mu \mathrm{m})$ & $R_{q}$ medio $(\mu \mathrm{m})$ & $R_{s k}$ medio & $R_{k u}$ medio \\
\hline $\begin{array}{l}\text { Nanocomposite } \\
\text { Mylar strip }\end{array}$ & Translúcido claro & $0,927 \pm 0,012$ & $1,156 \pm 0,015$ & $0,12 \pm 0,10$ & $3,4 \pm 0,3$ \\
\hline $\begin{array}{l}\text { Nanocomposite } \\
\text { Mylar strip }\end{array}$ & Esmalte A2 & $0,206 \pm 0,005$ & $0,258 \pm 0,004$ & $-0,124 \pm 0,023$ & $3,00 \pm 0,04$ \\
\hline $\begin{array}{c}\text { Composite supraesférico } \\
\text { Mylar strip }\end{array}$ & Translúcido & $0,322 \pm 0,004$ & $0,401 \pm 0,004$ & $-0,073 \pm 0,023$ & $2,92 \pm 0,03$ \\
\hline $\begin{array}{c}\text { Composite supraesférico } \\
\text { Mylar strip }\end{array}$ & Esmalte A2 & $0,174 \pm 0,005$ & $0,212 \pm 0,004$ & $-0,14 \pm 0,03$ & $3,28 \pm 0,09$ \\
\hline $\begin{array}{c}\text { Nanocomposite } \\
\text { OptiDisc } ®\end{array}$ & Translúcido claro & $2,43 \pm 0,06$ & $3,18 \pm 0,08$ & $-0,30 \pm 0,18$ & $3,66 \pm 0,20$ \\
\hline $\begin{array}{c}\text { Nanocomposite } \\
\text { OptiDisc } ®\end{array}$ & Esmalte A2 & $0,90 \pm 0,08$ & $1,24 \pm 0,13$ & $-1,61 \pm 0,23$ & $7,6 \pm 1,6$ \\
\hline $\begin{array}{c}\text { Composite supraesférico } \\
\text { OptiDisc } \AA\end{array}$ & Translúcido & $1,55 \pm 0,03$ & $2,07 \pm 0,04$ & $-0,59 \pm 0,09$ & $4,69 \pm 0,09$ \\
\hline $\begin{array}{c}\text { Composite supraesférico } \\
\text { OptiDisc } \AA\end{array}$ & Esmalte A2 & $0,339 \pm 0,008$ & $0,63 \pm 0,03$ & $-6,34 \pm 0,17$ & $71 \pm 3$ \\
\hline $\begin{array}{c}\text { Cerámica de circonia } \\
\text { sinterizada }\end{array}$ & Color SP3 & $1,295 \pm 0,014$ & $1,578 \pm 0,014$ & $-0,06 \pm 0,06$ & $2,49 \pm 0,07$ \\
\hline $\begin{array}{l}\text { Cerámica de circonia } \\
\text { sinterizada }\end{array}$ & Sin color & $1,268 \pm 0,017$ & $1,613 \pm 0,021$ & $-0,32 \pm 0,05$ & $3,29 \pm 0,12$ \\
\hline $\begin{array}{c}\text { Cerámica de circonia } \\
\text { presinterizada }\end{array}$ & - & $1,024 \pm 0,016$ & $1,264 \pm 0,015$ & $-0,28 \pm 0,04$ & $2,41 \pm 0,03$ \\
\hline
\end{tabular}


Aunque los valores de $R_{a}$ y $R_{q}$ aumentan considerablemente con el tratamiento superficial, las observaciones anteriores son válidas para los composites con y sin tratamiento. En cambio, el comportamiento de los parámetros $R_{s k}$ y $R_{k u}$ no es el mismo. Para los especímenes sin tratamiento, los valores de Rsk tienden a 0 y los de se $R_{k u}$ aproximan a 3, por lo que las superficies presentan, aproximadamente, una distribución normal de alturas. Con el tratamiento superficial, los valores de $R_{s k}$ disminuyen (se hacen más negativos) y los de $R_{k u}$ aumentan, alejándose de 0 y 3 respectivamente. Como consecuencia, puede concluirse que el tratamiento superficial aplicado a las resinas de composite no solo incrementa la rugosidad media de la superficie sino que también afecta a la simetría y a la forma de la distribución de alturas, alejándola de la distribución normal.

Para las cerámicas de circonia, las diferencias existentes entre los dos colores no son tan significativas como las existentes entre las distintas tonalidades de los composites. Los parámetros de rugosidad $R_{a}$ y $R_{q}$ presentan valores inferiores para la cerámica presinterizada. Por otro lado, los parámetros $R_{s k}$ y $R_{k u}$ en las muestras sinterizadas y la presinterizada toman valores similares, con $R_{s k}$ próximo a 0 y $R_{k u}$ a 3 . Por lo tanto, para las cerámicas de circonia analizadas, puede afirmarse que el proceso de sinterización resulta en un aumento de la rugosidad media sin afectar significativamente a la forma y la simetría de la distribución de alturas de la superficie.

En la Tabla III, se cuantifican las diferencias relativas en los parámetros de rugosidad debidas al tratamiento superficial de los composites y al proceso de sinterización de las cerámicas de circonia. Las incertidumbres asociadas se determinaron mediante propagación cuadrática de incertidumbres [18]. Nótese que, como consecuencia de dicha propagación de incertidumbres, las precisiones correspondientes a cada muestra son diferentes.

Cabe destacar que los valores de las incertidumbres encontradas permiten comparar y distinguir las características de la superficie de los biomateriales analizados.

\section{Conclusiones}

La evaluación de las características de la superficie es de gran interés para las aplicaciones clínicas de los biomateriales. La estructura superficial puede caracterizarse mediante el cálculo de parámetros de naturaleza estadística.

TABLA III

Diferencias relativas en los valores medios de los parámetros de rugosidad debidas al tratamiento superficial de los composites y al proceso de sinterización de las cerámicas de circonia, con sus correspondientes incertidumbres.

\begin{tabular}{|c|c|c|c|c|}
\hline Biomaterial & $\begin{array}{c}\text { Diferencia relativa en } \\
R_{a}(\mu \mathrm{m}) \\
\end{array}$ & $\begin{array}{c}\text { Diferencia relativa en } \\
R_{q}(\mu \mathrm{m}) \\
\end{array}$ & $\begin{array}{c}\text { Diferencia } \\
\text { relativa en } R_{s k} \\
\end{array}$ & $\begin{array}{c}\text { Diferencia } \\
\text { relativa en } R_{k u} \\
\end{array}$ \\
\hline $\begin{array}{l}\text { Nanocomposite } \\
\text { Translúcido claro }\end{array}$ & $1,62 \pm 0,08$ & $1,75 \pm 0,08$ & $3 \pm 3$ & $0,08 \pm 0,10$ \\
\hline $\begin{array}{c}\text { Nanocomposite } \\
\text { Esmalte A2 }\end{array}$ & $3,4 \pm 0,4$ & $3,8 \pm 0,5$ & $12 \pm 3$ & $1,5 \pm 0,5$ \\
\hline $\begin{array}{c}\text { Composite supraesférico } \\
\text { Translúcido }\end{array}$ & $3,82 \pm 0,11$ & $4,17 \pm 0,13$ & $7 \pm 3$ & $0,60 \pm 0,04$ \\
\hline $\begin{array}{c}\text { Composite supraesférico } \\
\text { Esmalte A2 }\end{array}$ & $0,96 \pm 0,07$ & $1,96 \pm 0,14$ & $45 \pm 10$ & $20,8 \pm 1,2$ \\
\hline $\begin{array}{c}\text { Cerámica de circonia } \\
\text { Color SP3 }\end{array}$ & $0,265 \pm 0,024$ & $0,249 \pm 0,018$ & $0,79 \pm 0,22$ & $0,03 \pm 0,03$ \\
\hline $\begin{array}{l}\text { Cerámica de circonia } \\
\text { Sin color }\end{array}$ & $0,24 \pm 0,03$ & $0,277 \pm 0,023$ & $0,15 \pm 0,25$ & $0,37 \pm 0,05$ \\
\hline
\end{tabular}


En este trabajo se han evaluado los principales parámetros de rugosidad $\left(R_{a}, R_{q}, R_{s k}\right.$ y $\left.R_{k u}\right)$ en distintos tipos de resinas de composite y cerámicas de circonia, proporcionando información, no sólo sobre la rugosidad media, sino también sobre la simetría y la forma de la distribución superficial de alturas. A fin de comparar los distintos biomateriales, se determinaron también las incertidumbres asociadas a los parámetros de la superficie. Para ello se empleó un sistema no invasivo basado en triangulación óptica: el microtopógrafo laser MICROTOP.06.MFC. Los resultados confirman la idoneidad de este sistema para obtener los principales parámetros de rugosidad con una precisión tal que permita establecer comparaciones entre distintos materiales y entre un mismo material en diferentes condiciones (tratamiento superficial en composites y proceso de sinterización en cerámica de circonia).

Los parámetros de rugosidad $R_{a}$ y $R_{q}$ muestran diferencias notables entre las distintas tonalidades de cada tipo de composite pero no entre los diferentes colores de las cerámicas de circonia sinterizadas.

Comparando los distintos tipos de resinas dentales de un mismo shade se encuentra que el nanocomposite presenta mayores valores de $R_{a}$ y $R_{q}$, lo cual puede estar relacionado con la formación de cúmulos de mayor tamaño en este tipo de composite.

Los resultados indican que el tratamiento superficial aplicado a los composites no solo aumenta su rugosidad sino que modifica la distribución superficial de alturas, alejándola de la distribución normal. En cambio, el proceso de sinterización de la cerámica de circonia hace que aumente la rugosidad media prácticamente sin afectar a la simetría y a la forma de la distribución de alturas de la superficie. De ello se deduce que, para que un análisis de dichos biomateriales tenga sentido comparativo se necesita el estudio, no sólo de $R_{a}$ y $R_{q}$, sino también de los parámetros skewness y kurtosis.

\section{Agradecimientos}

Esta investigación ha sido parcialmente financiada por el Ministerio de Ciencia e Innovación a través del proyecto MAT200909795 y la beca FPU concedida a Alicia Fernández-Oliveras. 